\title{
A influência dos papéis sociais na qualidade de vida de portadores de esquizofrenia
}

\author{
Tatiane Grigolatto ${ }^{a}$, Marcos Hortes Nisihara Chagas ${ }^{a}$, Clarissa Trzesniak ${ }^{a}$, \\ José Alexandre de Souza Crippa ${ }^{\mathrm{b}}$, Jaime Eduardo Cecílio Hallak ${ }^{\mathrm{b}}$, Jair Lício Ferreira Santos ${ }^{\mathrm{c}}$ \\ aPrograma de Pós-Graduação em Saúde Mental, Departamento de Neurociências e Ciências do Comportamento, \\ Faculdade de Medicina de Ribeirão Preto - FMRP, Universidade de São Paulo - USP, Ribeirão Preto, SP, Brasil

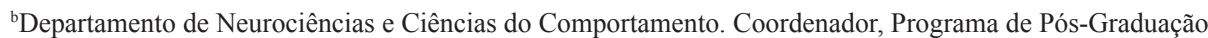 \\ em Saúde Mental, Departamento de Neurociências e Ciências do Comportamento, Faculdade de Medicina de \\ Ribeirão Preto - FMRP, Universidade de São Paulo - USP, Ribeirão Preto, SP, Brasil

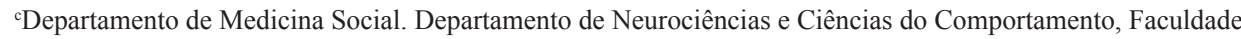 \\ de Medicina de Ribeirão Preto - FMRP, Universidade de São Paulo - USP, Ribeirão Preto, SP, Brasil
}

\begin{abstract}
Resumo: Introdução: Considerando o prejuízo causado pela esquizofrenia e a tendência mundial de inserir e tratar seus portadores na comunidade, torna-se imprescindível rever a literatura científica referente aos fatores influentes associados à qualidade de vida dessa população. Método: Uma revisão bibliográfica foi realizada com o objetivo de descrever quais papéis sociais influenciam na qualidade de vida e quais variáveis foram investigadas. Resultados: Foram selecionados 17 estudos, a maior parte de países europeus. A maioria foi realizada após o ano 2000. Quase todos os estudos eram seccionais e a maior parte avaliou amostras ambulatoriais. De um modo geral, a maioria dos estudos relatou uma melhor qualidade de vida associada com maior número de papéis sociais. Os papéis sociais apareceram atrelados aos domínios da qualidade de vida e nenhum dos estudos utilizou um instrumento específico para papéis sociais. Conclusões: A necessidade de maior número de estudos com aprofundamento sobre os aspectos centrais do tema se fez notar, devido à importância percebida sobre a influência dos papéis sociais na qualidade de vida.
\end{abstract}

Palavras-chave: Esquizofrenia, Qualidade de Vida, Revisão de Literatura.

\section{The influence of social roles in the quality of life of patients with schizophrenia}

\begin{abstract}
Introduction: Considering the damage caused by schizophrenia and the world tendency to treat and include its patients in the community, it is essential to review the scientific literature concerning the influential factors associated with quality of life in this population. Method: A literature review was carried out aiming to describe which social roles influence the quality of life and which variables have been investigated. Results: We selected 17 studies, mostly from European countries, conducted after 2000. Almost all studies were of the cross-sectional type and assessed outpatient samples. In general, most studies reported a better quality of life associated with a greater number of social roles. The social roles appeared linked to the areas of quality of life and none of the studies used a specific instrument for social roles. Conclusions: The review reveals the necessity for more studies regarding quality of life and social roles in schizophrenia.
\end{abstract}

Keywords: Schizophrenia, Quality of Life, Literature Review.

Autor para correspondência: Tatiane Grigolatto, Departamento de Neurociências e Ciências do Comportamento, Hospital das Clínicas, Faculdade de Medicina de Ribeirão Preto, Universidade de São Paulo, Av. dos Bandeirantes, 3900, $3^{\circ}$ andar, CEP 14025-048, Ribeirão Preto, SP, Brasil, e-mail: tgrigolatto@uol.com.br

Recebido em 16/1/2012; Revisão em 12/6/2012; Aceito em 13/7/2012. 


\section{Introdução}

De acordo com a Organização Mundial da Saúde OMS (2009), a esquizofrenia afeta aproximadamente sete em cada mil adultos. A maioria apresenta os primeiros sinais do transtorno entre 15 e 35 anos e embora a incidência seja baixa, a prevalência é alta devido à cronicidade. Esse transtorno danifica o funcionamento social, afetivo e produtivo do portador (SHIRAKAWA, 2009).

Indivíduos com esquizofrenia vivendo na comunidade têm necessidades adicionais. Portadores sintomáticos, por exemplo, precisam, na maior parte dos casos, da permanência constante em tratamento especializado (KATSCHNIG, 1997). Esses, muitas vezes estão sujeitos a diversas formas de preconceito $\mathrm{e}$ têm que enfrentar o estigma associado ao transtorno mental. Dessa forma, os usuários da saúde mental com esquizofrenia podem ter oportunidades sociais e econômicas limitadas e apresentar qualidade de vida prejudicada.

O conceito de qualidade de vida da Organização Mundial da Saúde (OMS) é o mais aceito e utilizado pela comunidade científica. Para essa organização,

[...] qualidade de vida é a percepção do indivíduo de sua posição na vida, de acordo com o contexto cultural e o sistema de valores nos quais vive e em relação aos seus objetivos, expectativas, padrōes e preocupaçôes. (WHOQOL GROUP, 1995, p. 3).

O interesse pela qualidade de vida de indivíduos com esquizofrenia teve início com o retorno de portadores de transtornos mentais crônicos à comunidade, decorrente do movimento de desinstitucionalização ocorrido nos países desenvolvidos ocidentais nos anos 1960 e 1970. Demandas como segurança pessoal, pobreza e isolamento social tornaram-se preocupaçóes dos usuários da saúde mental egressos de hospitais psiquiátricos, de seus familiares, profissionais de saúde e gestores de saúde (AWAD; VORUGANTI; HESLEGRAVE, 1997). Seguindo esse preceito, as pesquisas sobre aspectos psicossociais da esquizofrenia vêm aumentando com os anos.

Lehman, Ward e Linn (1982) expóem que o conhecimento sobre a qualidade de vida dos usuários da saúde mental pode auxiliar na compreensão a respeito da transformação que esses transtornos provocam globalmente na vida e no funcionamento das pessoas acometidas, bem como sobre a influência da assistência à saúde sobre seu bem-estar geral. Em se tratando de doenças crônicas, como no caso dos transtornos mentais graves e persistentes, a qualidade de vida torna-se ainda mais importante, já que o tratamento não é curativo. Dessa forma, o tratamento de portadores de transtornos mentais graves considera não apenas a redução de sintomas, mas enfatiza também a melhora no funcionamento social e no desempenho de papéis (DRAKE et al., 2003).

Papéis são as orientaçôes dadas às pessoas para que ajam de forma compatível com suas funções sociais, como, por exemplo, estudante, trabalhador ou pai (BARRETT; KIELHOFNER, 2002). Esse conceito está bem estabelecido em ciências sociais, antropologia, sociologia e psicologia (KAHN; ANTONUCCI, 1979). Comunidades, grupos e famílias, entre outros, são contextos para o desempenho de papéis, portanto, a vida do indivíduo pode ser estruturada em torno das funçóes que ele tem ou almeja. Assim, a função é definida como um conjunto de atividades que se espera de uma pessoa em virtude de seu papel e implica em uma determinada posição no espaço social.

Kahn e Antonucci (1979) ressaltam a importância das funções que são esperadas de uma pessoa e como essas nos ajudam a entender as mudanças que ocorrem no curso da vida, pois todos nós ocupamos determinada posição no espaço social. Embora uma pessoa possa sustentar diversos papéis ao longo da vida, alguns estão explicitamente relacionados à idade, principalmente quanto à hierarquia (KAHN; ANTONUCCI, 1979). Dessa forma, o desenvolvimento implica, necessariamente, na mudança de papéis. Essa mudança, por sua vez, depende do funcionamento adaptativo individual. De acordo com esses autores, os papéis apresentam características positivas ao favorecerem oportunidades de aquisição e uso de habilidades. Por outro lado, apresentam também características negativas ao gerarem tensóes, conflitos e ambiguidades (KAHN; ANTONUCCI, 1979).

Considerando o prejuízo causado pela esquizofrenia na aquisição de papéis e que a incapacidade para o desempenho desses, geralmente, antecede a internação psiquiátrica (PARSONS, 1958 apud OAKLEY; KIELHOFNER; BARRIS, 1985), fator que contraria a tendência mundial de inserir e tratar seus portadores na comunidade, podemos inferir que a qualidade de vida também pode ser afetada, portanto torna-se imprescindível rever a literatura científica referente aos fatores associados a ela junto a essa população. Optamos por enfatizar os papéis sociais por causa da importância desses para o desenvolvimento humano e pela escassez de estudos sobre o tema. 


\section{Método}

Uma revisão sistemática da literatura científica foi conduzida nas bases de dados Web of Science, LILACS, SciELO, Psychoinfo e PubMed, utilizando como palavras-chave: "qualidade de vida", "esquizofrenia", "papéis sociais", "quality of life", "social role" e "schizophrenia". Foram encontrados 449 artigos e selecionados 17, de acordo com os critérios de inclusão. Esses foram: participantes com esquizofrenia ou outros transtornos que se enquadrem no espectro da esquizofrenia (como transtorno esquizofreniforme ou transtorno esquizotípico), sem limite de tempo, em inglês, português e espanhol. Sem restrição quanto ao tipo de desenho de estudo e incluindo artigos que exploraram os fatores sociodemográficos, os aspectos clínicos (sintomas positivos, negativos e depressivo-ansiosos) e os papéis sociais. O impacto do uso de medicaçóes antipsicóticas sobre os papéis sociais e a qualidade de vida foi excluído dessa revisão, já que a extensão e a complexidade envolvidas demandariam uma revisão exclusiva.

\section{Resultados}

A maioria dos estudos foi realizada após o ano 2000. Todos foram seccionais, com exceção de dois (DIXON et al., 2001; TEMPIER; MERCIER; LEOUFFRE, 1997). A maior parte avaliou amostras de indivíduos em acompanhamento ambulatorial (GROZNIK; TAVCAR; LICINA, 2001; PIRFO et al., 1994; HALFORD; HAYES, 1995; FRANZ et al., 2000; DIXON et al., 2001; SÖRGAARD et al., 2002; CARDOSO et al., 2005, 2006; SUNG; PUSKAR, 2006). Os instrumentos mais utilizados para avaliar a qualidade de vida foram o Perfil de Qualidade de Vida Lancashire (LQoLP) e a Escala de Qualidade de vida (QLS) (Tabela 1).

\section{Qualidade de vida e fatores clínico-demográficos}

\subsection{Gênero e qualidade de vida}

A associação entre gênero e qualidade de vida foi significativa em quatro estudos (GROZNIK; TAVCAR; LICINA, 2001; SÖRGAARD et al., 2002; CARDOSO et al., 2005, 2006). Desses, todos relataram uma pior qualidade de vida entre os homens esquizofrênicos quando comparados com as mulheres e dois estudos destacavam itens isolados em que as mulheres se saiam melhor (CARDOSO et al., 2005, 2006).
Em geral, as mulheres têm apresentaçóes mais brandas de esquizofrenia, com início tardio e mais oportunidades de concluir os estudos e estabelecer mais relaçóes interpessoais (GROZNIK; TAVCAR; LICINA, 2001; SÖRGAARD et al., 2002). Aparentemente, essas diferenças têm impacto sobre as condições objetivas de qualidade de vida desses indivíduos. Cardoso et al. $(2005,2006)$ observaram que havia uma proporção maior de casados entre as mulheres esquizofrênicas em relação aos homens. $\mathrm{O}$ impacto do gênero foi marcante nesse estudo, pois as mulheres, independente de seu estado conjugal, apresentam-se mais satisfeitas, com melhor integração social, interpessoal, com satisfação em atividades de lazer e com sentimento de bem-estar. Outros estudos não encontraram relaçáo entre gênero e qualidade de vida na esquizofrenia (KUGO et al., 2006; MEIJER et al., 2009).

\subsection{Duração da doença, idade e qualidade de vida}

A idade parece não exercer grande efeito sobre a qualidade de vida de portadores de esquizofrenia (TEMPIER; MERCIER; LEOUFFRE, 1997; SÖRGAARD et al., 2002; KUGO et al., 2006; MEIJER et al., 2009). Entretanto, um estudo observou uma pior qualidade de vida em portadores de esquizofrenia mais velhos (CARDOSO et al., 2006). Segundo alguns autores, o período da vida em que a doença se manifesta traz maior ou menor prejuízo para os portadores (GROZNIK; TAVCAR; LICINA, 2001; CARDOSO et al., 2005).

Dois estudos evidenciaram uma correlação entre tempo de doença e qualidade de vida: quanto mais anos de doença, menor a qualidade de vida (CARDOSO et al., 2005; HANSSON et al., 1999). Por outro lado, dois estudos não encontraram relação significativa (KUGO et al., 2006; MEIJER et al., 2009).

\subsection{Escolaridade, renda e qualidade de vida}

Praticamente nenhum dos estudos (TEMPIER; MERCIER; LEOUFFRE, 1997; PIRFO et al., 1994; HALFORD; HAYES, 1995; FRANZ et al., 2000; DIXON et al., 2001; SÖRGAARD et al., 2002; SUNG; PUSKAR, 2006; KUGO et al., 2006; HANSSON et al., 1999; BELLACK et al., 1990; BREKKE et al., 1993; ATKINSON et al., 1996; CORRING; COOK, 2007) investigou a associação entre escolaridade e qualidade de vida em indivíduos esquizofrênicos. Contudo, uma pior qualidade de 


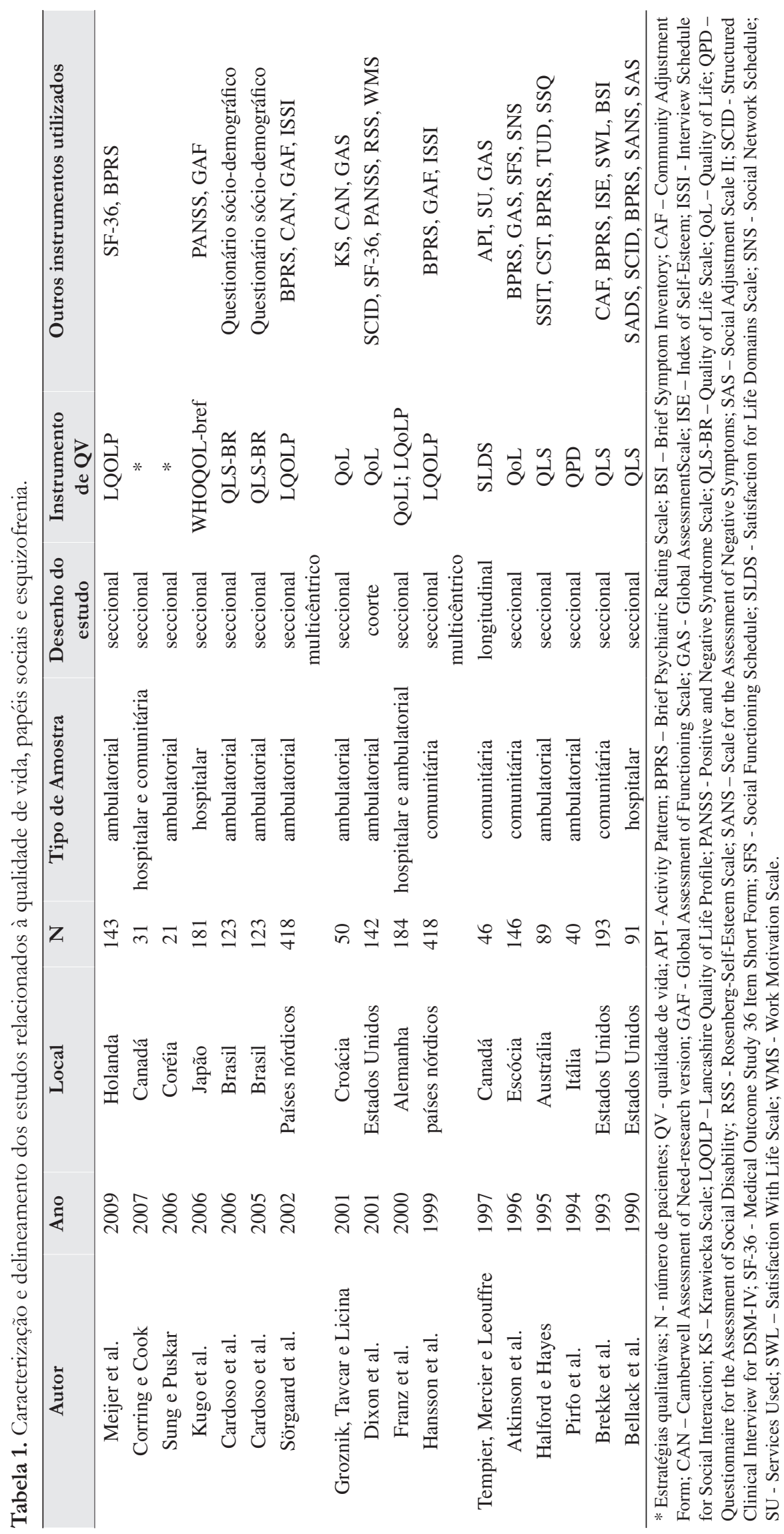


vida entre as pessoas com esquizofrenia com menor escolaridade foi investigada e observada em dois estudos (GROZNIK; TAVCAR; LICINA, 2001; CARDOSO et al., 2005).

A renda foi investigada por seis estudos, sendo que em cinco a renda baixa foi atrelada à pior qualidade de vida (TEMPIER; MERCIER; LEOUFFRE, 1997; CARDOSO et al., 2005; MEIJER et al., 2009; HANSSON et al., 1999; CORRING; COOK, 2007) e em um estudo essa correlação não foi significativa (DIXON et al., 2001).

\subsection{Moradia e qualidade de vida}

A moradia apareceu como variável influenciadora da qualidade de vida em cinco estudos (TEMPIER; MERCIER; LEOUFFRE, 1997; SÖRGAARD et al., 2002; CARDOSO et al., 2005; HANSSON et al., 1999; BREKKE et al., 1993). Entretanto, em um único estudo o tipo de moradia foi especificado e nele a localização urbana aparece relacionada a melhor qualidade de vida (SÖRGAARD et al., 2002). Seguindo outra linha, Cardoso et al. (2006) destacaram que a importância consiste no número de pessoas, sendo que um maior número de pessoas diminui a qualidade de vida.

\subsection{Variáveis clínicas e qualidade de vida}

Os sintomas depressivos, de forma independente do instrumento utilizado para mensurá-los, mostraram-se associados a uma pior qualidade de vida em indivíduos esquizofrênicos (SÖRGAARD et al., 2002; KUGO et al., 2006; MEIJER et al., 2009; HANSSON et al., 1999). Os sintomas ansiosos também se mostraram associados a uma pior qualidade de vida de usuários da saúde mental esquizofrênicos em alguns estudos (SÖRGAARD et al., 2002; MEIJER et al., 2009; HANSSON et al., 1999).

Os sintomas positivos da esquizofrenia mostraram-se associados a uma pior qualidade de vida nesses indivíduos em dois estudos (GROZNIK; TAVCAR; LICINA, 2001; CORRING; COOK, 2007). Outros estudos (HALFORD; HAYES, 1995; HANSSON et al., 1999) não confirmaram essa associação. Um estudo evidenciou associação entre sintomas negativos e uma pior qualidade de vida (BELLACK et al., 1990). Contudo, assim como no caso dos sintomas positivos, outros estudos não encontraram essa associação (HALFORD; HAYES, 1995; KUGO et al., 2006). Finalmente, alguns estudos reconhecem a influência dos sintomas na qualidade de vida dos usuários da saúde mental, porém não classificam esses sintomas em positivos e negativos (GROZNIK; TAVCAR; LICINA, 2001; DIXON et al., 2001; SUNG; PUSKAR, 2006). Cardoso et al. (2005) citaram a agitação psicomotora como um fator preditivo para baixa qualidade de vida.

\section{Relação entre qualidade de vida e papéis sociais}

Todos os estudos selecionados relataram uma melhor qualidade de vida associada com a presença de papéis sociais. Os papéis sociais aparecem atrelados aos domínios da qualidade de vida e nenhum dos estudos utilizou um instrumento específico para papéis sociais. $\mathrm{O}$ aumento das limitações de papéis foi associado à redução da aceitação da medicação, à satisfação geral com a vida, saúde, atividades diárias e confiança (DIXON et al., 2001). Dois estudos evidenciam a importância dos papéis sociais para a qualidade de vida, onde os usuários da saúde mental revelam que o sentimento de perda de eficácia no desempenho dos papéis sociais diminui a qualidade de vida (SUNG; PUSKAR, 2006; CORRING; COOK, 2007).

\subsection{Papel social trabalhador}

Este é o mais citado, tanto como finalidade em intervençóes terapêuticas (PIRFO et al., 1994; ATKINSON et al., 1996), quanto como marcador de deterioração no funcionamento social (BELLACK et al., 1990; BREKKE et al., 1993). A presença do trabalho diminui o sofrimento intrapsíquico e aumenta a autoestima (BREKKE et al., 1993). O trabalho também aparece como um dos fatores que favorecem a qualidade de vida (SÖRGAARD et al., 2002; CARDOSO et al., 2005, 2006; SUNG; PUSKAR, 2006).

\subsection{Papel social amigo}

Foi observado que ter amigo próximo e ter grande número de amigos e parentes disponíveis na rede social estavam associados com melhora da qualidade de vida global (GROZNIK; TAVCAR; LICINA, 2001; MEIJER et al., 2009; HANSSON et al., 1999; ATKINSON et al., 1996; CORRING; COOK, 2007), assim como ser capaz de fazer outros amigos (SÖRGAARD et al., 2002). Da mesma forma, a solidão e dificuldade de fazer amizades repercutem negativamente na qualidade de vida (TEMPIER; MERCIER; LEOUFFRE, 1997; SUNG; PUSKAR, 2006). 


\subsection{Papel social cônjuge}

Investigaçôes apontam o estado conjugal como preditor de melhor evoluçáo da esquizofrenia. Esse marcador é visto como indicador de competência social (CARDOSO et al., 2006). Alto funcionamento global e melhor integração social, como ser casado, foi medianamente relatado como preditor positivo de qualidade de vida (MEIJER et al., 2009). O estado civil solteiro teve três vezes mais chances de ser associado com baixos níveis de qualidade de vida em comparação aos casados (CARDOSO et al., 2005). Para homens, ser solteiro apresenta dez vezes mais chances de estar em uma categoria de menor qualidade de vida quando comparado aos casados. Entre as mulheres, ser solteira apresenta 4,5 vezes mais chances de estar em uma categoria de menor qualidade de vida quando comparada com as casadas (CARDOSO et al., 2006).

\subsection{Papel social estudante}

O papel de estudante foi abordado no estudo de Sung e Puskar (2006), que mostrou que os estudantes tinham dificuldade de concentração, de compreensão de leitura, de fazer provas e completar suas grades. Apenas alguns estudantes relataram sentimentos positivos em relaçáo à escola e desejo de continuar a busca acadêmica (SUNG; PUSKAR, 2006). A escola pode ser um contexto de desempenho favorável à medida que estimula o funcionamento social. Com o aumento do funcionamento social, o sofrimento intrapsíquico diminui, a autoestima aumenta e, finalmente, quando o funcionamento social aumenta a satisfação com a vida também cresce (BREKKE et al., 1993). A maioria dos estudantes participantes relataram mais experiências negativas do que positivas atreladas a esse papel, como perda de interesse na vida e perda de sentimentos de realidade em atividades de vida diária (SUNG; PUSKAR, 2006).

\subsection{Papel social religiosidade e de voluntário}

A importância da espiritualidade para a qualidade de vida foi enfatizada pelos participantes nos estudos de Hansson et al. (1999) e Corring e Cook (2007). Entretanto, nenhum desses se aprofundou no tema. Outro papel que também foi apenas citado é o de voluntário, ele aparece como forma de trabalho, juntamente com serviço doméstico, de uma pequena parcela $(4 \%)$ dos indivíduos avaliados (BREKKE et al., 1993).

\subsection{Outros achados relevantes}

No estudo de Sung e Puskar (2006), todos os participantes relataram mais de uma experiência negativa sobre sua doença mental. Alguns aceitaram sua doença e acrescentaram uma nova identidade, a de usuário da saúde mental doente mental (SUNG; PUSKAR, 2006). Um dos fatores que influenciam negativamente a qualidade de vida é o medo dos sintomas retornarem (CORRING; COOK, 2007). A internalização do papel de usuário da saúde mental parece facilitar o tratamento e a aceitação da doença.

No estudo realizado por Tempier, Mercier e Leouffre (1997), os usuários da saúde mental avaliaram as atividades na comunidade como menos satisfatórias do que no passado. O funcionamento global dos usuários da saúde mental diminuiu ao longo do tempo, apesar de a pontuaçáo na qualidade de vida ter sido mantida (TEMPIER; MERCIER; LEOUFFRE, 1997). Os déficits nas habilidades sociais contribuem para um baixo funcionamento social do usuário da saúde mental com esquizofrenia (HALFORD; HAYES, 1995).

Habilidades sociais são reflexo do desenvolvimento de papéis, aparentando ter um efeito significante sobre o funcionamento social na comunidade, independentemente de outros sintomas (BELLACK et al., 1990). As habilidades sociais e o funcionamento social na comunidade são relacionados, independentemente da intensidade de sintomas psicóticos (HALFORD; HAYES, 1995). Essas revisôes acusam a estreita ligação entre papéis sociais e qualidade de vida. Os papéis sociais, por sua vez, envolvem tarefas sociais e sugerem que essas tarefas medem diferentes habilidades.

\section{Discussão}

Existem dúvidas quanto à capacidade do usuário da saúde mental com esquizofrenia avaliar sua própria qualidade de vida devido à sintomatologia, déficit cognitivo e/ou falta de conhecimento sobre a morbidade. Entretanto, Awad, Voruganti e Heslegrave (1997) assinalaram que muitos estudos têm confirmado a coerência e confiabilidade de relatos feitos por esquizofrênicos sobre seu nível de satisfação interna e sentimentos.

Em relação às variáveis clínicas não houve um consenso entre os estudos. Os sintomas depressivos, de forma independente do instrumento utilizado para mensurá-los, mostraram-se associados a uma pior qualidade de vida em usuários da saúde mental esquizofrênicos (HANSSON et al., 1999; SÖRGAARD et al., 2002; KUGO et al., 2006; MEIJER et al., 2009). Os sintomas ansiosos também se mostraram associados a uma pior qualidade de vida de usuários da saúde mental esquizofrênicos 
em alguns estudos (HANSSON et al., 1999; SÖRGAARD et al., 2002; MEIJER et al., 2009).

Os sintomas positivos da esquizofrenia mostraram-se associados a uma pior qualidade de vida em usuários da saúde mental esquizofrênicos em dois estudos (GROZNIK; TAVCAR; LICINA, 2001; CORRING; COOK, 2007). Porém outros estudos não confirmaram essa associação (HALFORD; HAYES, 1995; HANSSON et al., 1999). Apenas um estudo evidenciou associação entre sintomas negativos e uma pior qualidade de vida entre esquizofrênicos (BELLACK et al., 1990). Contudo, assim como no caso dos sintomas positivos, outros estudos não encontraram essa associação (HALFORD; HAYES, 1995; KUGO et al., 2006). Finalmente, alguns estudos reconhecem a influência dos sintomas na qualidade de vida dos usuários da saúde mental, como o estudo de Cardoso et al. (2005), que cita a agitaçáo psicomotora como um fator preditivo para baixa qualidade de vida, outros estudos também reconhecem a importância, mas não classificam esses sintomas em positivos e negativos (GROZNIK; TAVCAR; LICINA, 2001; DIXON et al., 2001; SUNG; PUSKAR, 2006).

Um grande número de sintomas não impede, necessariamente, a melhora na função (DIXON et al., 2001). O aumento dos sintomas se relacionou com rede social pequena e diminuição das taxas de satisfação com a segurança pessoal (CORRING; COOK, 2007). A saúde física e as limitaçóes físicas não tiveram impacto sobre o trabalho, motivaçáo ou autopercepção da capacidade de trabalho (DIXON et al., 2001).

As mulheres, em geral, têm apresentaçôes mais brandas de esquizofrenia, com início tardio e mais oportunidades de concluir os estudos e estabelecer mais relaçóes interpessoais (GROZNIK; TAVCAR; LICINA, 2001; SÖRGAARD et al., 2002). Essas diferenças, aparentemente, têm impacto sobre as condiçóes objetivas de qualidade de vida desses indivíduos. Cardoso et al. (2005, 2006) observaram que havia uma proporção maior de casados entre as mulheres esquizofrênicas em relação aos homens. O impacto do gênero sobre a qualidade de vida foi marcante nesse estudo, pois as mulheres, independentemente de seu estado conjugal, apresentaram-se mais satisfeitas, com melhor integração social e interpessoal, com satisfação em atividades de lazer e com sentimento de bem-estar. Outros estudos não encontraram relação entre gênero e qualidade de vida na esquizofrenia (KUGO et al., 2006; MEIJER et al., 2009).

A idade parece não exercer grande efeito sobre a qualidade de vida do usuário da saúde mental esquizofrênico (TEMPIER; MERCIER; LEOUFFRE, 1997; SÖRGAARD et al., 2002;
KUGO et al., 2006; MEIJER et al., 2009). Entretanto, um estudo observou uma pior qualidade de vida em usuários da saúde mental mais velhos (CARDOSO et al., 2006). Segundo alguns autores, o período da vida em que a doença se manifesta traz maior ou menor prejuízo para os portadores (GROZNIK; TAVCAR; LICINA, 2001; CARDOSO et al., 2005). Dois estudos evidenciaram uma correlação entre tempo de doença e qualidade de vida, sendo que quanto mais anos de doença, menor a qualidade de vida (HANSSON et al., 1999; CARDOSO et al., 2006).

Outros fatores sociodemográficos como renda, escolaridade e condiçóes de moradia aparecem relacionados à qualidade de vida em alguns estudos. A renda foi investigada por seis estudos, sendo que em cinco desses a renda baixa foi atrelada a pior qualidade de vida (TEMPIER; MERCIER; LEOUFFRE, 1997; HANSSON et al., 1999; CARDOSO et al., 2005; CORRING; COOK, 2007; MEIJER et al., 2009) e em um estudo essa correlação não foi significativa (DIXON et al., 2001).

As condiçôes de moradia parecem influenciar a qualidade de vida, entretanto não houve um consenso entre os estudos que abordaram essa variável, pois em um estudo a residência urbana aparece como fator positivo (SÖRGAARD et al., 2002), em três estudos o tipo de residência não é especificado (BREKKE et al., 1993; TEMPIER; MERCIER; LEOUFFRE, 1997; HANSSON et al., 1999) e outro estudo destaca que a importância consiste em com quem se mora e no número de pessoas, sendo que quanto mais pessoas habitam a mesma residência, menor a qualidade de vida (CARDOSO et al., 2006). Em relação à escolaridade, somente dois estudos observaram que menor escolaridade é igual à menor qualidade de vida (GROZNIK; TAVCAR; LICINA, 2001; CARDOSO et al., 2005).

Grande parte dos estudos não avaliou a relação entre fatores sociodemográficos e esquizofrenia (BELLACK et al., 1990; PIRFO et al., 1994; HALFORD; HAYES, 1995; ATKINSON et al., 1996; FRANZ et al., 2000; SUNG; PUSKAR, 2006). No entanto, o papel das características sociodemográficas associado com qualidade de vida em usuário da saúde mental com esquizofrenia se mostrou importante.

Outras variáveis se fizeram notar como fatores preditivos para baixa qualidade de vida, entre elas o uso atual de três ou mais medicamentos psicoativos, tratamento ambulatorial atual (CARDOSO et al., 2005) e estigma, tanto auto quanto heteroestigma (CORRING; COOK, 2007). Dentro dos diferentes grupos de variáveis, os fatores preditivos positivos foram afeto (subjetivo), satisfação com relações sociais, relaçóes de suporte, ocupaçóes significativas, 
segurança pessoal, atividades de lazer, satisfaçáo com atividades diárias, alta autoestima e um positivo senso de autoconfiança, que levam a um aumento da qualidade de vida (HANSSON et al., 1999; SÖRGAARD et al., 2002; CORRING; COOK, 2007; MEIJER et al., 2009).

Esses resultados sugerem que, na esquizofrenia, embora as diferentes áreas de influencia estejam interligadas, as disfunçóes podem operar de forma independente e, portanto, têm diferentes fatores preditivos. Para além das manifestaçōes patológicas ocasionadas pela esquizofrenia, investigar a qualidade de vida desses usuários da saúde mental nos leva a pensar em quais aspectos encontramos saúde, quais papéis produtivos se mantiveram e quais necessitam de estímulo para progredir, não apenas para que esses usuários da saúde mental sejam menos onerosos para a sociedade, mas principalmente para que eles tenham a chance de escolher e experimentar papéis de vida significativos.

\section{Conclusão}

Qualidade de vida é um conceito individual, volúvel e complexo que difere de cultura para cultura, de época para época e de indivíduo para indivíduo. Ele se relaciona com os papéis que cada pessoa deseja para si própria. No entanto, sofre influência das expectativas que a sociedade tem em relação à contribuição que o indivíduo fornece para o todo; portanto, o conceito se modifica ao longo do tempo. De forma resumida, qualidade de vida é viver bem consigo mesmo e com outros que fazem parte das suas relaçóes tanto pessoais quanto profissionais. Tamanha particularidade em um conceito resultou no esforço de diversos autores para a criação de escalas de avaliação de qualidade de vida específicas para indivíduos com o mesmo diagnóstico médico. Adicionalmente, o fato de os critérios serem valorizados singularmente levou ao surgimento de diversas significaçóes para o termo.

É importante notar que quando pensamos sobre qualidade de vida existe uma diferença entre os conceitos e a forma subjetiva com que os usuários da saúde mental os experimentam e interpretam. Portanto, é difícil decidir quais fatores afetam a qualidade de vida subjetiva desses usuários da saúde mental. Cada pessoa coloca maior ou menor importância em uma das áreas citadas, dependendo da situação individual e da incumbência designada às inúmeras variáveis.

A necessidade de maior número de estudos com aprofundamento sobre os aspectos centrais do tema se fez notar, devido à importância da percepçáo da influência dos papéis sociais na qualidade de vida. As características sociodemográficas parecem contextualizar a relaçáo entre os papéis sociais mais prevalentes e sua influência na qualidade de vida, como no caso do papel social de estudante que foi investigado ricamente no estudo de Sung e Puskar (2006), em que os autores enfatizam a importância desse papel na sociedade coreana. Além da escassez de investigaçóes, papéis sociais como usuário da saúde mental e voluntário foram citados em apenas um estudo, outros, como paternidade/maternidade, não foram mencionados.

Outra deficiência percebida nesta revisão foi a falta, apesar de sua importância, de estudos longitudinais para investigar a perda e a manutenção de papéis para esses indivíduos e também quais os fatores os influenciam.

\section{Referências}

ATKINSON, J. M. et al. The impact of education groups for people with schizophrenia on social functioning and quality of life. British Journal of Psychiatry, London, v. 168, n. 2, p. 199-204, 1996. PMid:8837910. http:// dx.doi.org/10.1192/bjp.168.2.199

AWAD, A. G.; VORUGANTI, L. N. P.; HESLEGRAVE, R. J. Measuring quality of life in patients with schizophrenia. Pharmacoeconomics, v. 11, p. 32-47, 1997. PMid:10172917. http://dx.doi.org/10.2165/00019053-199711010-00005

BARRETT, L.; KIELHOFNER, G. Teorias Derivadas de Perspectivas do Comportamento Ocupacional. In: NEISTADT, M. E.; CREPEAU, E. B. (Org.). Willard \& Spakman - Terapia Ocupacional. 9. ed. Rio de Janeiro: Guanabara Koogan, 2002. p. 488-497.

BELLACK, A. S. et al. An analysis of social competence in schizophrenia. British Journal of Psychiatry, London, v. 156, p. 809-818, 1990. PMid:2207511. http://dx.doi. org/10.1192/bjp.156.6.809

BREKKE, J. S. et al. Psychosocial functioning and subjective experience in schizophrenia. Schizophrenia Bulletin, Rockville, v. 19, n. 3, p. 599-608, 1993. PMid:8235461. http://dx.doi.org/10.1093/schbul/19.3.599 CARDOSO, C. S. et al. Factors associated with low quality of life in schizophrenia. Caderno de Saúde Pública, Rio de Janeiro, v. 21, n. 5, p. 1338-1348, 2005.

CARDOSO, C. S. et al. Qualidade de vida e dimensão ocupacional na esquizofrenia: uma comparaçáo por sexo. Caderno de Saúde Pública, Rio de Janeiro, v. 22, n. 6, p. 1303-1314, 2006.

CORRING, D. J.; COOK, J. V. Use of qualitative methods to explore the quality-of-life construct from a consumer perspective. Psychiatric Services, Washington, v. 58, n. 2, p. 240-4, 2007. PMid:17287382.

DIXON, L. et al. The impact of health status on work, symptoms, and functional outcomes in severe mental illness. Journal of Nervous and Mental Disease, Baltimore, v. 189, n. 1, p. 17-23, 2001. PMid:11206660. http://dx.doi. org/10.1097/00005053-200101000-00004

DRAKE, R. E. et al. The history of community mental health treatment and rehabilitation for persons with severe mental illness. Community Mental Health Journal, 
New York, v. 39, n. 5, p. 427-40, 2003. PMid:14635985. http://dx.doi.org/10.1023/A:1025860919277

FRANZ, M. et al. The importance of social comparisons for high levels of subjective quality of life in chronic schizophrenic patients. Quality of Life Research, Dordrecht, v. 9, n. 5, p. 481-489, 2000.

GROZNIK, M.; TAVCAR, R.; LICINA, M. Outpatients with schizophrenia: Quality of life, needs and global assessment of efficiency and role functioning. Psychiatria Danubina, Zagreb, v. 13, n. 14, p. 15-20, 2001.

HALFORD, W. K.; HAYES, R. L. Social skills in schizophrenia - assessing the relationship between social skills, psychopathology and community functioning. Social Psychiatry and Psychiatric Epidemiology, Berlin, v. 30, n. 1, p. 14-19, 1995. PMid:7892613. http://dx.doi. org/10.1007/BF00784429

HANSSON, L. et al. Predictors of subjective quality of life in schizophrenic patients living in the community. A Nordic multicentre study. International Journal of Social Psychiatry, London, v. 45, n. 4, p. 247-258, 1999. PMid:10689608. http://dx.doi.org/10.1177/002076409904500403

KAHN, R. L.; ANTONUCCI, T. C. Convoys over the life course: Attachement, roles and support. Michigan: Institute for Social Research, University of Michigan, 1979.

KATSCHNIG, H. How useful is the concept of quality of life in psychiatry? Current Opinion in Psychiatry, Nova York, v. 10, n. 5, p.337-45, 1997. http://dx.doi. org/10.1097/00001504-199709000-00001

KUGO, A. et al. Quality of life for patients with schizophrenia in a Japanese psychiatric hospital. Psychiatry Research, Amsterdam, v. 144, n. 1, p. 49-56, 2006. PMid:16919759. http://dx.doi.org/10.1016/j. psychres.2005.06.012

LEHMAN, A. F.; WARD, N. C.; LINN, L. S. Chronic mental patients: The quality of life issue. American Journal of Psychiatry, Washington, v. 139, n. 10, p. 1271-1276, 1982.

MEIJER, C. J. et al. Predictors of general quality of life and the mediating role of health related quality of life in patients with schizophrenia. Social Psychiatry and Psychiatric Epidemiology, Berlin, v. 44, n. 5, p. 361-368, 2009. PMid:18974910. http://dx.doi.org/10.1007/ s00127-008-0448-4

OAKLEY, F.; KIELHOFNER, G.; BARRIS, R. An occupational therapy approach to assessing psychiatric patients' adaptive functioning. American Journal of Occupational Therapy, New York, v. 39, n. 3, p. 147-54 1985. PMid:3993720. http://dx.doi.org/10.5014/ajot.39.3.147 ORGANIZAÇÃO MUNDIAL DA SAÚDE - OMS. Esquizofrenia. 2009. Disponivel em: <http://www.who. int/schizophrenia>. Acesso em: 23 fev. 2010.

PIRFO, E. et al. Job preparation and improvement of the quality of life of schizophrenic patients in today's metropolis. International Journal of Mental Health, White Plains, v. 23, n. 1, p. 11-22, 1994.

SHIRAKAWA, I. Ajustamento social na esquizofrenia. 4. ed. São Paulo: Casa Leitura Médica, 2009.

SÖRGAARD, K. W. et al. Self-esteem in persons with schizophrenia. A Nordic multicentre study. Journal of Mental Health, Abingdon, v. 11, n. 4, p. 405-415, 2002. http://dx.doi.org/10.1080/09638230020023769

SUNG, K. M.; PUSKAR, K. R. Schizophrenia in college students in Korea: a qualitative perspective. Perspectives in Psychiatric Care, Hillsdale, v. 42, n. 1, p. 21-32, 2006. PMid:16480414. http://dx.doi. org/10.1111/j.1744-6163.2006.00046.x

TEMPIER, R.; MERCIER, C.; LEOUFFRE, P. Quality of life and social integration of severely mentally ill patients: A longitudinal study. Journal of Psychiatry of Neuroscience, Ottawa, v. 22, n. 4, p. 249-255, 1997. PMid:9262047 PMCid:PMC1188866.

WHOQOL GROUP. The World Health Organization Quality of Life Assessment (WHOQOL): position paper from the World Health Organization. Social Science of Medicine, Oxford, v. 41, p. 1403-10, 1995. http://dx.doi. org/10.1016/0277-9536(95)00112-K

\section{Contribuição dos Autores}

Tatiane Grigolatto realizou a revisão bibliográfica e escreveu o artigo. Marcos Hortes Nisihara Chagas ajudou a escrever o artigo. Clarissa Trzesniak fez a revisão de texto. José Alexandre de Souza Crippa ajudou a revisar o texto. Jaime Eduardo Cecílio Hallak foi co-orientador do trabalho. Jair Lício Ferreira Santos foi o orientador do trabalho. 